\title{
Steady-state pulse component in ultrafast pulse propagation in an anomalously dispersive dielectric
}

\author{
Heejeong Jeong* and Ulf L. Österberg \\ Thayer School of Engineering, Dartmouth College, Hanover, New Hampshire 03755, USA
}

(Received 12 August 2007; published 21 February 2008)

\begin{abstract}
We describe a theoretical method to distinguish between the precursors (transient part) and main signal (steady state) for a Gaussian pulse propagating through an anomalously dispersive linear dielectric. According to conventional theory of optical precursors, there is no main signal for Gaussian pulse propagation regardless of the system parameters. In this paper, we show the existence of the main pulse by comparing the conventional asymptotic theory with recently published analytical expressions. Additionally, we show that experimentally observed, asymmetrically extended tails in Gaussian pulse propagation are due to a resonant precursor.
\end{abstract}

DOI: 10.1103/PhysRevA.77.021803

PACS number(s): 42.25.Bs, 42.50.Gy, 42.50.Nn

Commercially available femtosecond lasers allow researchers to investigate ultrafast interactions between electromagnetic pulses and different types of media. For linear systems, the interactions can be divided into free (transient) and forced (steady-state) responses. A classical approach to describe weak optical pulse propagation in a dielectric medium leads to the concept of precursors (transients) and a main pulse (steady state). Historically, the first system studied analytically was a step-modulated pulse with carrier frequency $\omega_{c}$ propagating in a single-resonant Lorentz medium defined by a resonant frequency $\omega_{0}\left(\omega_{c} \neq \omega_{0}\right)$ and a spectral width $\delta\left(\delta \approx 0.1 \omega_{0}\right)[1,2]$. This artificial system had the advantage of clearly defining what we mean by precursors and a main pulse, but the disadvantage of not being easily generated in the laboratory. In general, experimental work is performed using pulses that can be well approximated as Gaussians. Chu and Wong were among the first to investigate optical Gaussian pulse propagation using thin samples of GaP:N [3]. Their experimental results compared favorably to the theory by Garrett and McCumber [4]. A more detailed analysis of Chu and Wong's experiments was performed by Tanaka et al. [5] using a saddle-point analysis. This work provided further insight into the physics of both "fast" and "slow" light for a Gaussian pulse propagating through an anomalously dispersive dielectric medium. Additional theoretical studies of Gaussian pulses have been done by Sauter [6] using the fast Fourier transform (FFT) algorithm, and by Oughstun and co-workers [7] using advanced asymptotic methods.

An important issue concerning Gaussian pulses arises when it is combined with conventional precursor theory. As stated by Oughstun [7]: “...the propagated field can be expressed solely in terms of a generalized Sommerfeld and a generalized Brillouin precursor field...." His point is well taken for short pulses, where transient effects are dominant. This is, however, hard to accept for a temporally long Gaussian pulse, because under such conditions it is difficult to generate a significant transient effect (precursors). In such a case, the steady-state part (main signal) ought to be dominant in the transmitted field. Furthermore, as shown in recent

\footnotetext{
*hjjeong@dartmouth.edu
}

studies (both experimental and theoretical) [8,9], the main signal (steady state) does exist together with transients for short time scale. Thus, the complete absence of the main signal in the description of Gaussian pulse propagation is unacceptable and needs to be investigated further.

We begin with a brief introduction of conventional asymptotic analysis. In general, the transmitted pulse in a medium at a distance $z$ can be described using a Fourier integral,

$$
E(z, t)=\frac{1}{2 \pi} \int_{-\infty}^{\infty} E(0, \omega) e^{(z / c) \phi(\omega, \theta)} d \omega
$$

where $E(0, \omega)$ is the spectrum of the initial pulse, the phase of the integrand $\phi(\omega, \theta)=i \omega[n(\omega)-\theta]=i c[k(\omega) z-\omega t] / z$, and $n(\omega)=\sqrt{1-\omega_{p}^{2} /\left(\omega^{2}-\omega_{0}^{2}+2 i \delta \omega\right)}$ is the index of refraction, $\omega_{p}$ is the plasma frequency, and $k(\omega)=\omega n(\omega) / c$ is the complex wave number. Asymptotically, as $z / c \rightarrow \infty$, Eq. (1) can be solved by the "method of steepest descent" [14]. In asymptotic analysis, precursors are evaluated by the contour integral near the saddle points of the phase $\phi(\omega, \theta)$, and the main signal is obtained by the residue of the pole at the carrier frequency $\omega_{c}$. For example, $E(0, \omega)$ $=i /\left[\sqrt{2 \pi}\left(\omega-\omega_{c}\right)\right]$, the step-modulated input spectrum has a pole at $\omega_{c}$. In the case of a Gaussian, however, there is no pole, implying the absence of the main signal.

In this paper, we limit our analysis to a weakly dispersive $\left(\omega_{p} \ll \omega_{0}\right)$ and narrow absorptive medium $\left(\delta \ll \omega_{0}, \omega_{c} \sim \omega_{0}\right)$ to make the mathematics more tractable and the physics more obvious. We begin by considering a short square pulse to show the existence of a main signal based on recent studies [8,9], see Fig. 1. Besides, considering a pulse with a rise time shorter than the medium's relaxation time, any pulse shape can be approximated as a square pulse. To define the main signal in the specific case of a Gaussian pulse, we consider the "modified" asymptotic analysis by Tanaka $[5,7]$, instead of using the conventional definition, Eq. (1). The Tanaka method provides three different field components; two transients (free) and one steady-state (forced) part. We also identify the experimentally observed extended tail of a transmitted Gaussian pulse [5,10-12] as precursors. This precursor analysis agrees with Hartmann and Laubereau's ear- 


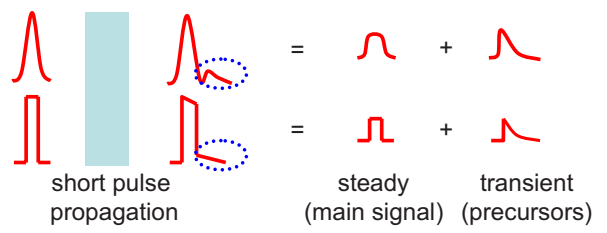

(a)
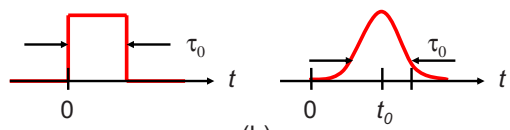

(b)

FIG. 1. (Color online) Illustration of a short pulse propagation. Gaussian vs square pulse. (a) Field components in total transmission. (b) The definition of pulse width.

lier statement [13] that the lagging asymmetric tail can be used to determine the dephasing time $T_{2}$ for a medium.

The recent studies [8,9] of optical precursors for a step pulse, $E(0, t)=\Theta(t) e^{-i \omega_{c} t}$, shows the instantaneous arrival of a main signal $E_{C}(z, \tau)$ together with the total precursors $E_{S B}(z, \tau)$ immediately after the front of the pulse. Note, if the main signal arrives within the short pulse duration, then we have the main signal component within the total transmission, i.e., it is very difficult to separate the transients from the main signal in the detected light. For the square input pulse $E(0, t)=\left[\Theta(t)-\Theta\left(t-\tau_{0}\right)\right] e^{-i \omega_{c} t}$, the total transmitted field is $E(z, \tau)=A(z, \tau) e^{-i \omega_{c} \tau}=E_{S B}(z, \tau)+E_{C}(z, \tau)$, where the total precursor is $E_{S B}(z, \tau)=A_{S B}(z, \tau) e^{-i \omega_{c} \tau}=A_{S B}(z, \tau) e^{-i \Delta \omega \tau} e^{-i \omega_{0} \tau}$, and the main signal is $E_{C}(z, \tau)=A_{C}(z, \tau) e^{-i \omega_{c} \tau}$, where the amplitudes are

$$
\begin{gathered}
A_{C}(z, \tau)=E_{0}\left[\Theta(\tau)-\Theta\left(\tau-\tau_{0}\right)\right] e^{-p / \Delta} \\
A_{S B}(z, \tau)=-E_{0} e^{-\Delta \tau} \sum_{n=1}^{\infty}\left(\Theta(\tau) \frac{J_{n}(2 \sqrt{p \tau})}{\tau^{n / 2}}\right. \\
\left.-e^{\tau_{0} \Delta} \Theta\left(\tau-\tau_{0}\right) \frac{J_{n}\left[2 \sqrt{p\left(\tau-\tau_{0}\right)}\right]}{\left(\tau-\tau_{0}\right)^{n / 2}}\right)\left(\frac{-\sqrt{p}}{\Delta}\right)^{n},
\end{gathered}
$$

for $\tau>p /|\Delta|^{2}$ in the first series, and $\left(\tau-\tau_{0}\right)>p /|\Delta|^{2}$ in the second series.

$$
\begin{aligned}
A_{S B}(z, \tau)= & E_{0} e^{-\Delta \tau} \sum_{n=0}^{\infty}\left(\Theta(\tau) \frac{J_{n}(2 \sqrt{p \tau})}{\tau^{-n / 2}}\right. \\
& \left.-e^{\tau_{0} \Delta} \Theta\left(\tau-\tau_{0}\right) \frac{J_{n}\left[2 \sqrt{p\left(\tau-\tau_{0}\right)}\right]}{\left(\tau-\tau_{0}\right)^{-n / 2}}\right)\left(\frac{\Delta}{\sqrt{p}}\right)^{n}-A_{C}(z, \tau),
\end{aligned}
$$

for $\tau<p /|\Delta|^{2}$ in the first series, and $\left(\tau-\tau_{0}\right)<p /|\Delta|^{2}$ in the second series, where the retarded time is $\tau \equiv t-z / c$, pulse width is $\tau_{0}$, the complex detuning is $\Delta \equiv-i\left(\omega_{c}-\omega_{0}\right)+\delta$, and $p \equiv \omega_{p}^{2} z /(4 c)=\alpha_{0} z \delta / 2$.

Figure 2(a) shows a reduced main signal by a factor of $e^{-p / \Delta}$ as in Eq. (2) for different carrier frequencies $\omega_{c}$ together with the reference (step response, dashed lines). Fig- (a)

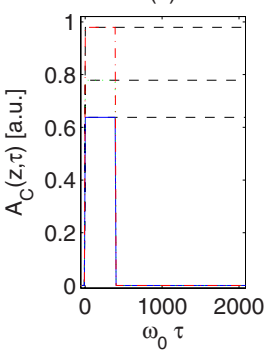

(d)
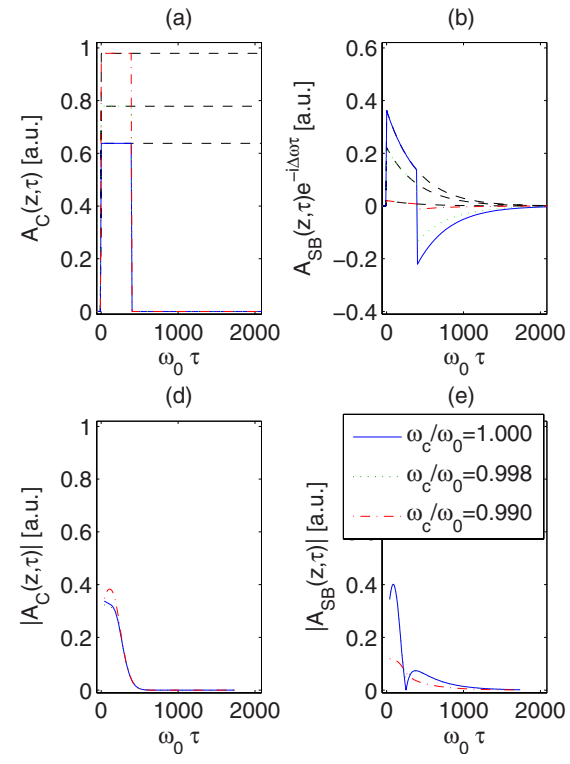

(c)

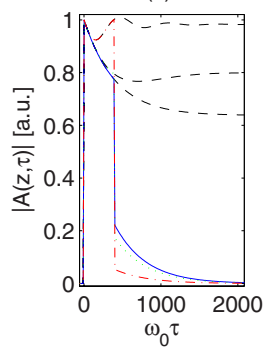

(f)

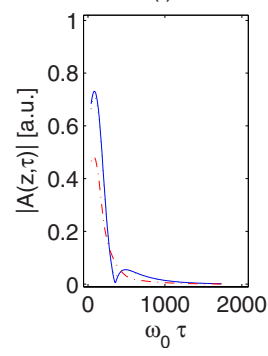

FIG. 2. (Color online) Main signal, precursors, and total amplitudes of square pulse transmission (a),(b),(c), and Gaussian pulse (d),(e),(f). (a) Main signal amplitudes oscillating at $\omega_{c}$ for different carrier frequencies $\omega_{c} / \omega_{0}=1\left(\omega_{c}=\omega_{0}\right), \omega_{c} / \omega_{0}=0.998\left(\omega_{c}=\omega_{0}-\delta\right)$, $\omega_{c} / \omega_{0}=0.990\left(\omega_{c}=\omega_{0}-5 \delta\right)$, (b) precursor amplitudes oscillating at $\omega_{0}$, and (c) total amplitude based on analytic expressions in Eqs. (2)-(4) together with the reference (step-response [9], dashed lines). For the Gaussian, (d) main signal, (e) precursors, and (f) total amplitude are evaluated by the method of steepest descent, Eq. (7). Parameters are $z / z_{0}=1.18 \times 10^{7}, \omega_{p} / \omega_{0}=1.74 \times 10^{-5}, \delta / \omega_{0}=0.002$, and $\omega_{0} \tau_{0}=392.31$.

ure 2(b) shows the corresponding transient, amplitudes oscillating at $\omega_{0}$, decaying exponentially as $e^{-\delta \tau}$, which is analogous to the results in $[8,9]$. The decay continues with a sudden $\pi$-phase shift, when the external light is turned off, due to the remnant medium polarization as described by Crisp [16]. Before the "turn off," the modulation of the total square pulse transmission [Fig. 2(c)] is due to the difference between the carrier frequency $\omega_{c}$ and the saddle-point frequency $\omega_{s p}$, as recently observed in [9]. In the case of a narrow absorption band, the saddle-point frequency $\omega_{s p}$ moves very quickly towards the resonance frequency $\omega_{0}$, and can therefore be approximated as $\omega_{s p} \approx \omega_{0}$ for most of the transient time period $[8,9]$. If we instead consider a Gaussian pulse with very fast rise and fall times, close to that of the square pulse, the main signal and total precursors should be similar to the results in Figs. 2(d)-2(f). Due to the absence of an analytic expression for the Gaussian pulse, we use a numerical asymptotic analysis.

A realistic definition of optical precursors and main signal for Gaussian pulse propagation is achieved by using the modified phase introduced by Tanaka et al. [5] and Oughstun and co-workers [7]. Both introduced the normalized distance $z / z_{0}$, where $z_{0}=\lambda_{0} / 2 \pi=c / \omega_{0}$, so that the asymptotic theory can be used for small $z$ as long as $z / z_{0}$ is large compared to the medium's characteristic wavelength, $\lambda_{0}$. For a transformlimited Gaussian input pulse $E(z=0, t)=E_{0} e^{-4\left(t-t_{0}\right)^{2} / \tau_{0}^{2}} e^{-i \omega_{c} t}$ (Fig. 1), the input spectrum is Gaussian as well, 
(a)

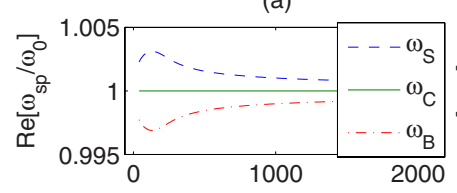

(b)

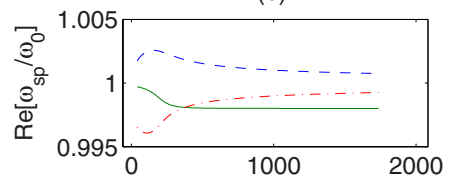

(c)

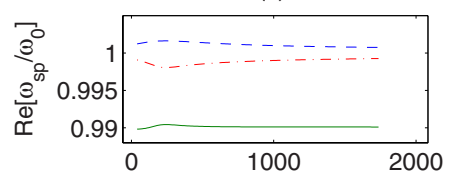

(d)
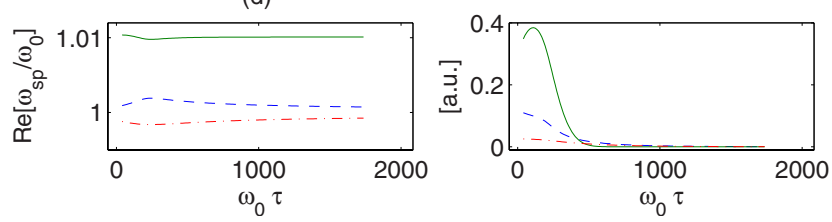

FIG. 3. (Color online) Numerically evaluated saddle points and corresponding field components. The real part of saddle points $\omega_{s p}$ and absolute field envelope $\left|A_{\omega_{s p}}(z, \tau)\right|$ for different carrier frequencies $\omega_{c}$ (a) $\omega_{c} / \omega_{0}=1\left(\omega_{c}=\omega_{0}\right)$, (b) $\omega_{c} / \omega_{0}=0.998\left(\omega_{c}=\omega_{0}-\delta\right)$, (c) $\omega_{c} / \omega_{0}=0.99 \quad\left(\omega_{c}=\omega_{0}-5 \delta\right)$, and (d) $\omega_{c} / \omega_{0}=1.01 \quad\left(\omega_{c}=\omega_{0}+\delta\right)$, using $z / z_{0}=1.18 \times 10^{7}, \quad \omega_{p} / \omega_{0}=1.74 \times 10^{-5}, \quad \delta / \omega_{0}=0.002$, and $\omega_{0} \tau_{0}=392.31$.

$$
E(0, \omega)=\frac{E_{0} \tau_{0}}{2 \sqrt{2}} e^{-\left(\tau_{0}^{2} / 16\right)\left(\omega-\omega_{c}\right)^{2}+i\left(\omega-\omega_{c}\right) t_{0}},
$$

where $t_{0}$ is the time for the pulse to have the maximum value. We let $t_{0}=1.51743 \tau_{0}$ based on a noise level of $10^{-4}$. When we put Eq. (5) into Eq. (1), the transmitted field associated with the method of steepest decent is given as

$$
\begin{aligned}
E(z, t) & =\frac{E_{0} \tau_{0} e^{-i \omega_{c} t_{0}}}{4 \sqrt{\pi}} \int_{-\infty}^{\infty} e^{\left(z / z_{0}\right) \Phi(\omega, \theta)} d \omega \\
& =\sum_{\omega_{s p}}^{5} \frac{E_{0} \tau_{0} e^{-i \omega_{c} t_{0}}}{4 \sqrt{\pi}} \frac{e^{\left(z / z_{0}\right) \Phi\left(\omega_{s p}, \theta\right)-i \psi}}{\sqrt{\left(z / z_{0}\right)\left|\Phi^{\prime \prime}\left(\omega_{s p}, \theta\right)\right|}},
\end{aligned}
$$

where $\theta=c\left(t-t_{0}\right) / z, \psi$ is the angle of steepest decent path, and modified phase is

$$
\Phi(\omega, \theta)=\phi(\omega, \theta)-\frac{z_{0}}{z} \frac{\tau_{0}^{2}}{16}\left(\omega-\omega_{c}\right)^{2} .
$$

The modified phase, Eq. (8), has five saddle points satisfying $\partial_{\omega} \Phi\left(\omega_{s p}, \theta\right)=0$. As mentioned by Tanaka, only three saddle points contribute to the transmitted pulse. The behavior of the three saddle points are known for Brillouin's parameters $\left(\omega_{c} \neq \omega_{0}, \omega_{p} \sim \omega_{0}, \delta \sim 0.1 \omega_{0}\right)[5,7]$. But for such a strongly dispersive case, the main signal is totally absorbed and one is left with only the precursors. Thus, we use parameters favorable to detect the main signal [9], as used in Fig. 2: (a) $\omega_{c} / \omega_{0}=0.99$

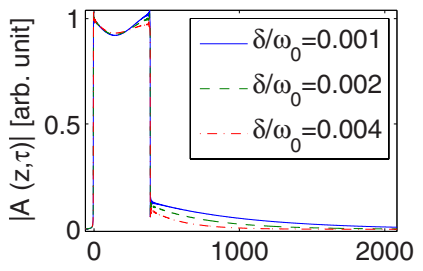

(c) $\omega_{c} / \omega_{0}=0.99$

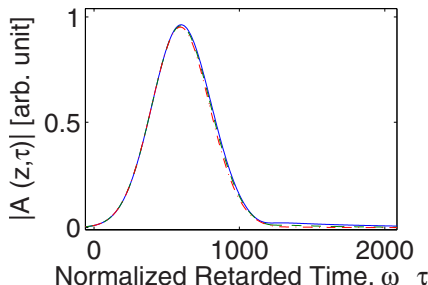

Normalized Retarded Time, $\omega_{0} \tau$ (b) $\omega_{c} / \omega_{0}=1$

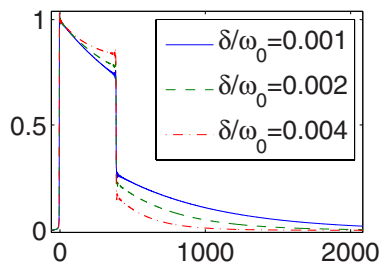

(d) $\omega_{c} / \omega_{0}=1$

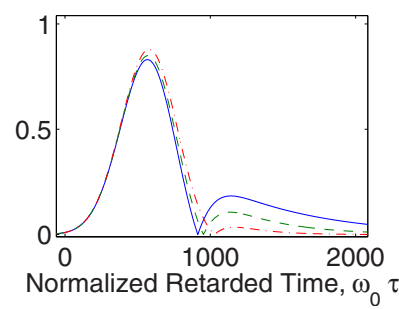

FIG. 4. (Color online) Gaussian vs square pulse propagation for different medium broadenings $\delta / \omega_{0}$, and for two different carrier frequencies $\omega_{c} / \omega_{0}$. (a) Square and (c) Gaussian pulse propagation for the off-resonance case: $\omega_{c} / \omega_{0}=0.99$. (b) Square and (d) Gaussian pulse for the on-resonance case: $\omega_{c} / \omega_{0}=1$, using $z / z_{0}$ $=1.18 \times 10^{7}, \omega_{p} / \omega_{0}=1.74 \times 10^{-5}$, and $\omega_{0} \tau_{0}=392.31$.

$z / z_{0}=1.18 \times 10^{7}, \quad \omega_{p} / \omega_{0}=1.74 \times 10^{-5}, \quad \delta / \omega_{0}=0.002, \quad$ and $\omega_{0} \tau_{0}=392.31$.

Figure 3 shows three saddle points and corresponding field envelopes for various input carrier frequencies $\omega_{c}$. The total transmitted pulse in Eq. (7) can be expressed as $E(z, \tau)=\sum_{\omega_{s p}}^{3} A_{\omega_{s p}}(z, \tau) e^{-i \omega_{s p} \tau}$. We denote the frequency component higher than $\omega_{0}$ as $S$ ("Sommerfeld"), the lower frequency component as $B$ ("Brillouin"), and the frequency close to the carrier $\left(\omega_{c}\right)$ as $C$ ("carrier" frequency of the main signal). The real part of the saddle points, $\operatorname{Re}\left(\omega_{s p} / \omega_{0}\right)$ in the right column is the frequency for each field. Note that, $\omega_{S}$ denoted as the dashed line $\left(\omega_{B}\right.$ denoted as semidashed line) moves towards $\omega_{0}$ from above (below) resonance frequency. The solid line denotes $\omega_{C}$, which is always stabilized around $\omega_{c}$ and behaves similarly to the frequency of the main signal for the "resonant precursor" of a step-modulated pulse [8,9], especially for $\omega_{c} / \omega_{0}=1$ in Fig. 3(a). A difference from the step-pulse case is the frequency chirp starting from a finite value instead of the infinite. In Fig. 3(b), $\omega_{B}$ shows an interesting transition from lower to higher frequency towards $\omega_{0}$ with respect to $\omega_{c}$. Before the transition, $\omega_{C}$ differs from $\omega_{c}$, which provides the parameter regime where the modified phase method fails to define the main signal.

For the absolute field envelopes, $\left|A_{\omega_{s p}}(z, \tau)\right|$ in the right column of Fig. 3, $\left|A_{\omega_{C}}(z, \tau)\right|$ drops within a fall time $[\sim 234$ in normalized units $\left.\left(\omega_{0} \tau\right)\right]$ after the "turn off." The transient parts, $\left|A_{\omega_{S}}(z, \tau)\right|$ and $\left|A_{\omega_{B}}(z, \tau)\right|$, decay exponentially similar to Fig. 2(b). Both amplitudes are equal for on-resonance case [Fig. 3(a)], but $A_{B}(z, \tau)$ dominates over $A_{S}(z, \tau)$ for the red detuning $\left(\omega_{c} / \omega_{0}=0.998\right.$, or 0.99$)$, and vice versa for blue detuning $\left(\omega_{c} / \omega_{0}=1.01\right)$.

It is not surprising to observe the transient in a ultrafast input pulse $\left(\omega_{0} \tau_{0}=392\right)$ compared to a longer medium relax- 
ation time (500 in normalized unit). A notable finding is the steady-state part $\left[A_{C}(z, \tau)\right.$ oscillating at $\omega_{C}=\omega_{c}$, solid line $]$ similar to the main signal in square pulse propagation. For the original phase $\phi(\omega, \theta)$ in Eq. (1), there are two saddle points out of four which contribute to the optical precursors. The information about the main signal is found in the Gaussian spectrum $E(0, \omega) \propto e^{-\tau_{0}^{2}\left(\omega-\omega_{c}\right)^{2} / 16+i\left(\omega-\omega_{c}\right) t_{0}}$ by considering the physical meaning of a pole as the primary source of the steady-state part. Thus, the modified phase $\Phi(\omega, \theta)$ including the Gaussian spectrum is crucial for recovering the main signal. Figures 2(d)-2(f) shows the main signal, total precursors, and total field envelopes, respectively, to be compared with the analytic expression for the square pulse [Figs. 2(a)-2(c)].

We also find that the asymmetric tails decay exponentially with time for both cases after turn off. The sudden $\pi$-phase shift manifests itself with a null for the Gaussian pulse. These transient tails are also observed to be more significant as we move closer to the absorption region, $\omega_{c} \approx \omega_{0}$, as shown in Figs. 4(b) and 4(d). Based on these observations, we argue that these tails are due to resonant precursors $[8,9,15]$. Also, the narrow medium resonance $\delta$ excites stronger transient tails than the broad absorption bands (Fig. 4). In other words, as the pulses become shorter compared to the medium's dephasing time $T_{2}=1 / \delta$, the transient effect becomes more dominant. This is why the transmitted energy of the short pulse decays less than exponentially, as also described in Crisp's quantum optical "small area pulse" [16]. For short square (or Gaussian) pulses, the transient response occurs after the turn off of the pulse, as observed by others $[5,7]$.

In summary, the square pulse approach allows us to use recent analytical methods $[8,9]$ to identify a main signal and precursors for Gaussian-like short pulse propagation through linear Lorentz dielectrics. The results of the square pulse qualitatively agrees with asymptotic theory with the modified phase for the Gaussian. This approach naturally includes the main pulse and also provides a physical explanation for the extended tail of a Gaussian pulse. The tail is a remnant free oscillation (precursors) after the forced oscillation of the medium is turned off.

We gratefully acknowledge discussions of this research with Professor Ursula Gibson and the financial support of NIST through Grant No. 60NANB4D1142.
[1] L. Brillouin, Wave Propagation and Group Velocity (Academic, New York, 1960).

[2] K. E. Oughstun and G. C. Sherman, Electromagnetic Pulse Propagation in Causal Dielectrics (Springer-Verlag, Berlin, 1994).

[3] S. Chu and S. Wong, Phys. Rev. Lett. 48, 738 (1982).

[4] C. G. B. Garrett and D. E. McCumber, Phys. Rev. A 1, 305 (1970).

[5] M. Tanaka, M. Fujiwara, and H. Ikegami, Phys. Rev. A 34, 4851 (1986).

[6] T. Sauter, J. Phys. A 35, 6743 (2002).

[7] C. M. Balictsis and K. E. Oughstun, Phys. Rev. E 47, 3645 (1993); K. E. Oughstun and C. M. Balictsis, Phys. Rev. Lett. 77, 2210 (1996); C. M. Balictsis and K. E. Oughstun, Phys. Rev. E 55, 1910 (1997); K. E. Oughstun and N. A. Cartwright, J. Mod. Opt. 52, 1089 (2005).
[8] William LeFew, Ph.D. thesis, Duke University, 2007.

[9] H. Jeong, A. M. C. Dawes, and D. J. Gauthier, Phys. Rev. Lett. 96, 143901 (2006); H. Jeong, Ph.D. thesis, Duke University, 2006.

[10] N. C. Nielsen et al., Phys. Rev. B 64, 245202 (2001).

[11] A. I. Talukder and M. Tomita, Phys. Rev. A 72, 051802(R) (2005).

[12] T. Kohmoto, Y. Fukui, S. Furue, K. Nakayama, and Y. Fukuda, Phys. Rev. E 74, 056603 (2006).

[13] H.-J. Hartmann and A. Laubereau, Opt. Commun. 47, 117 (1983).

[14] G. B. Arfken and H. J. Weber, Mathematical Methods for Physicists (Academic, San Diego, 1995).

[15] E. Varoquaux, G. A. Williams, and O. Avenel, Phys. Rev. B 34, 7617 (1986).

[16] M. D. Crisp, Phys. Rev. A 1, 1604 (1970). 